\title{
Electronic Report Generation Web Service evaluated within a Telemedicine System
}

\author{
I. Martínez-Sarriegui $^{1,3}$, M. E. Hernando ${ }^{1,3}$, F. J. Brito ${ }^{1}$, G. García-Sáez ${ }^{1,3}$, J. Molero ${ }^{1}$, M. Rigla ${ }^{2,3}$, \\ E. Brugués ${ }^{2,3}$, A. de Leiva ${ }^{2,3}$, E. J. Gómez ${ }^{1,3}$ \\ ${ }^{1}$ Bioengineering and Telemedicine Centre, Polytechnic University of Madrid, Spain \\ ${ }^{2}$ Endocrinology Department, Sant Pau Hospital, Barcelona, Spain \\ ${ }^{3}$ CIBER-BBN Networking Research Centre, Spain
}

\begin{abstract}
This work presents a generic tool based on a client-server architecture that generates electronic reports helping the evaluation process of any information system. For the specific evaluation of telemedicine systems the defined reports cover four dimensions: auditory of the system; evolution of clinical protocols; results from the questionnaires for user acceptance and quality of life; and surveillance of clinical variables. The use of a Web Service approach allows multiplatform use of the developed electronic report service and the modularity followed in the implementation enables easy system evolution and scalability.
\end{abstract}

Keywords- Web Service, Electronic Report, Evaluation, Surveillance.

\section{INTRODUCTION}

Telemedicine has shown to be a very useful tool to help chronic patients in the continuous control required by their diseases [1]. But not all of the telemedicine approaches are suitable for all the medical scenarios. A deep evaluation along the telemedicine clinical interventions is needed in order to measure both the impact in the patient care and the impact of the enhanced interactions between the principal actors of the system.

This work presents a generic tool based on a client-server architecture that generates electronic reports helping the evaluation process of any information system. For the specific evaluation of telemedicine systems the defined reports cover four dimensions: auditory of the system; evolution of clinical protocols; results from the questionnaires for user acceptance and quality of life; and surveillance of clinical variables.

The electronic reports are generated on PDF format. This format is accessible, secure, reliable, and the most important, it is an open format that allows information exchange.

Previous studies [2] have demonstrated that it is possible to improve patient's care and save time and space using electronic reports in contrast with traditional paper ones. The use of simple tools allows removing the principal barriers of Information Technology systems, that is the implementation of too complex tools that require technical knowledge from users [3][4].

The tool presented in this paper has been integrated and evaluated within DIABTel [5], a Telemedicine System for patients with type 1 diabetes that has a distributed multiaccess architecture based on software agents.

The persons suffering Type 1 diabetes are not able to produce insulin by themselves and they need external insulin administration to maintain blood glucose concentrations close to normoglycemia. These patients require a strict control of their diet, exercise and insulin injections to avoid severe, acute and long-term complications that can be responsible for premature death and disability [6]. The DIABTel system allows the registration of monitoring data and provides decision support tools for therapy adjustment. The system has supported several randomized clinical experiments [7]

\section{Material And Methods}

\section{A. Definition of the electronic reports}

Two types of reports have been defined depending on the nature of the information contained on them: 1) Intervention Reports to analyse a posteriori a clinical intervention, including patient's demographic data, use of the system, results of the subjective questionnaires and impact of the intervention in clinical parameters; 2) surveillance reports that presents, for a predefined time period, the statistics from monitoring data, information about downloadings from medical devices, calculus of risk indexes and warnings.

The content of Surveillance Reports can be personalized by the users. The reports defined by patients will be filled with their own information whereas reports defined by physicians summarize information from all the patients. Fig. 1 shows the different types of reports and the associated content.

Intervention Reports are referred to a specific randomized clinical intervention that is performed to a set of patients that have participated in the control and 
intervention group within two fixed dates. The Intervention Reports are generic reports and could be applied to other telemedicine interventions for different pathologies.

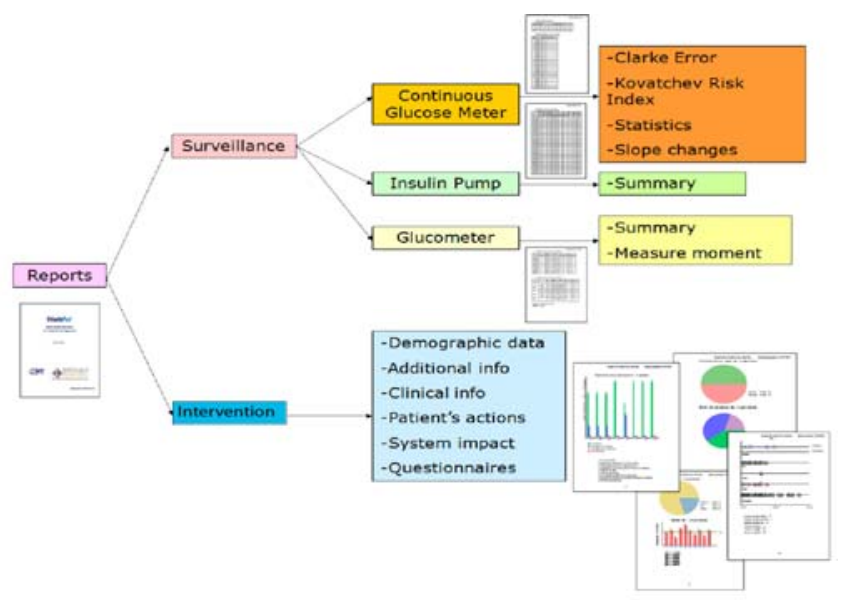

Fig. 1 Available reports and their content

Surveillance Reports are dependent on the specific disease, due to their link to medical variables and monitoring devices.

\section{B. Architecture of the system}

The architecture of the system, Fig. 2, is based on a client-server model. The role of server is played by the Report Server, which has been integrated as an agent within the DIABTel multi-access system (MAS). The role of the client is distributed among the users, when they access the tool that is embedded in DIABTel's Web interface, and among other applications that can request for reports at any time. The Web Service communicates client and server by exchanging specific messages, as it is specified on the standard [8]. The advantage of using this architecture is that applications developed with different programming languages and running in different platforms can communicate between them assuring interoperability.

The Report Server is continually waiting for report's requests. Clients connect to it and request a specific report. The server processes the request, creates the report and offers it to the client. In the DIABTel experience, some reports are generated on user demand and others are generated periodically when automatically requested by the Periodicity Agent according to a predefined periodicity configuration related to the request parameters and the frequency in the generation of a specific report (daily, weekly, monthly, etc.) Users just have to interact with the users' interface and the client logic processes the connections and requests in an automatic and transparent way.

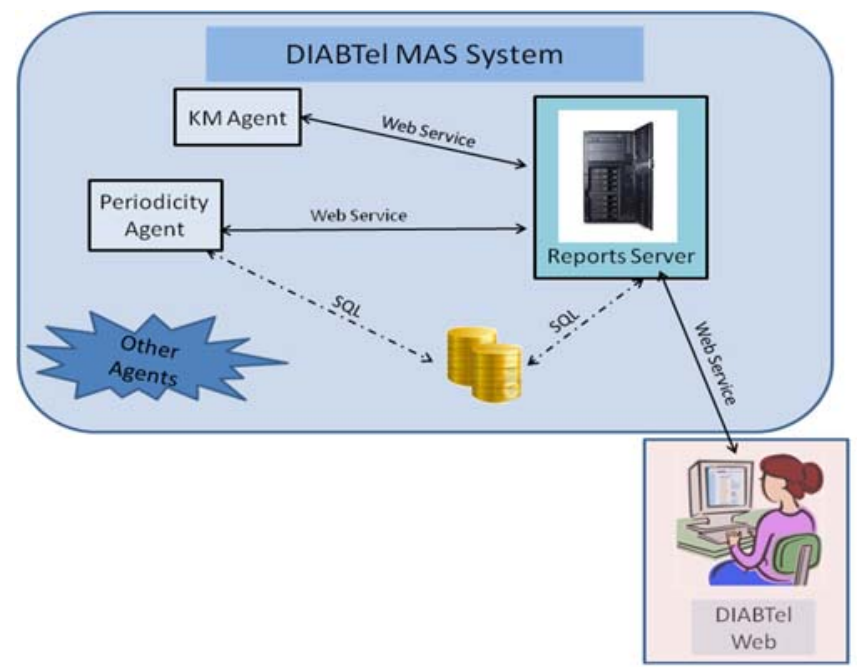

Fig. 2 System architecture

To create Intervention Reports, the Report Server obtains from the database all the information associated to the selected individuals for a given time period and condenses it in a set of graphics, allowing a synthesis of the information. Some predefined templates have been created to help users to choose the structure of the requested report. Thus, to obtain a report, users do choose a template which content is automatically filled with data from the selected clinical intervention and users can restrict the set of patients and the initial and final dates for the report. Physicians and system administrators can create, modify and delete templates.

Surveillance Reports present the processed data in tables that summarize relevant information to help patients and doctors to control the disease. Data processing is accomplished by an agent of the DIABTel system, the Knowledge Management Agent [9] (KM Agent), that analyses the data downloaded from patients' medical devices: insulin pump, continuous glucose meter and glucometer. With this information, the agent makes an automatic analysis, based on fuzzy logic, of the variables that represent the metabolic state of the patient.

The analysis of the data downloaded from medical devices is made every time a file from a device is attached to the system, through DIABTel's Web site, or every time any user of the system demands a surveillance report using the interface. When users request a surveillance report, they have to specify the affected user (patient), the type of report: pump, continuous glucose meter, glucometer or combined and the time span of the data. 
The KM Agent can be also activated by the Periodicity Agent. In this scenario, when the KM Agent finishes the data processing, it sends to the Report Server all the information that has to be shown in the tables.

\section{The Report Server}

The Report Server generates reports and manages report templates. It creates printable PDF files filled with information obtained from the database or directly from the $\mathrm{KM}$ Agent and stores them in the telemedicine system where they can be accessed by users through an URL that is linked in the Web interface.

\section{Client}

The criteria for the users' interface implementation have been to create an intuitive, accessible and easy to use interface where users have templates to configure the parameters of the desired report. The interface is based on forms where the user has to insert the search criterion parameters (template, clinical intervention, set of patients, initial and final dates). The interface informs users in case any mistake has been done. The developed interface is sensitive to the user's role (physician, system administrator or patient) offering only the functionalities that each user can use. The interface has been integrated in DIABTel's Web site following its look and feel design criteria.

\section{E. Architecture Evaluation}

In this paper we present a study of the features offered by the architecture described above. To perform the evaluation analysis we measured the file size and total reports' generation times for different types of reports associated to the clinical intervention described in [7]. All the reports cover the whole clinical intervention period (2 months).

To generate Intervention Reports the system needs to do SQL queries to the database but when creating the Surveillance Reports the KM Agent is who performs the SQL queries and provides all the information during the request. For this reason, a different analysis is made for each type of report.

For Intervention Reports, the generation time mainly depends on the following factors: 1 ) the connection with the Web Service to initiate a request; 2) the information volume presented on the report and the consequent database query time and PDF file size; 3) the URL return. For Surveillance Reports the predominant time is the communication with KM Agent and the time consumed by the agent processing the desired data.
The Minimum Intervention Report, with smallest generation time, is the one including just the demographic information (5 graphs) and the Maximum one, with the biggest generation time is a report with all the available information (34 graphs). The evaluated Surveillance Reports contain data from a single patient: insulin pump monitoring data, continuous glucose monitoring (CGM) and glucometer data.

We also studied the influence of increasing the information volume and the contribution of different factors in the reports' generation time

\section{RESUlts}

\section{A. Total reports' generation time}

Table 1 shows the results of total reports' generation time. Intervention Reports' generation time range between 1.5 seconds and 26 seconds and depends on the content of the report, that is, in the number of graphics represented on it, and in the time span selected for the data. As an example, a study Report summarizing a clinical experiment where 10 patients were followed-up during the 4 months takes 25 seconds (including demographic data, use of the system, patients' interactions with the system and the professionals, questionnaires results and lab tests). On the other hand, surveillance reports' generation time is nearly the same for all the reports: approximately 14.5 seconds, where CGM stands for continuous glucose meter.

Table 1 Reports' Generation Time

\begin{tabular}{lllllc}
\hline \multicolumn{2}{c}{ Type of report and content } & $\begin{array}{l}\text { Generation } \\
\text { time }\end{array}$ & $\begin{array}{l}\text { No. } \\
\text { Days }\end{array}$ & $\begin{array}{l}\text { File } \\
\text { Size }\end{array}$ & $\begin{array}{l}\text { No. } \\
\text { Graphs / } \\
\text { Tables }\end{array}$ \\
\hline Intervention & Minimum & 1.5 sec. & 60 & $70 \mathrm{kB}$ & 5 \\
Report & Maximum & 26 sec. & 60 & $500 \mathrm{kB}$ & 34 \\
& CGM & 14.50 sec. & 3 & $78 \mathrm{kB}$ & 5 \\
& CGM & 14.65 sec. & 30 & $120 \mathrm{kB}$ & 5 \\
& Insulin pump & $14.73 \mathrm{sec}$. & 380 & $193 \mathrm{kB}$ & 1 \\
Surveillance & Insulin pump & $15.22 \mathrm{sec}$. & 1275 & $273 \mathrm{kB}$ & 1 \\
Report & CGM + pump & $14.77 \mathrm{sec}$. & 51 & $91 \mathrm{kB}$ & 6 \\
& CGM + pump & $14.97 \mathrm{sec}$. & 730 & $197 \mathrm{kB}$ & 6 \\
& Glucometer & $14.66 \mathrm{sec}$. & 58 & $73 \mathrm{kB}$ & 2 \\
& Glucometer & $15.80 \mathrm{sec}$. & 381 & $578 \mathrm{kB}$ & 2 \\
\hline
\end{tabular}

Results show that generation time for surveillance reports does not depend on the size of the file or on the amount of information represented. 


\section{B. Contributions to reports' generation time}

Fig. 3 illustrates the contribution of an increase in the time span of the data represented in the graphs and tables of the reports. It can be seen that a big increase in the time span only contributes to a small increase in generation time. Comparing with Table 1, we can conclude that for Intervention Reports, the number of graphics to represent has a bigger influence in reports' generation time than the data time span.

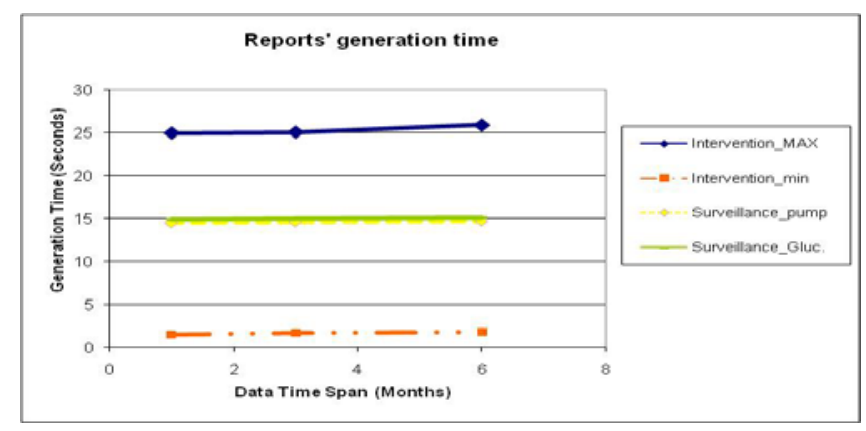

Fig. 3 Data time span-Reports' generation time dependence

Fig. 4 shows the different factors that contribute to the reports' generation time. For Intervention Reports the predominant contribution is the time used by the Report Server filling the content of the reports; and for Surveillance Reports the predominant contribution is the data processing time in KM Agent.

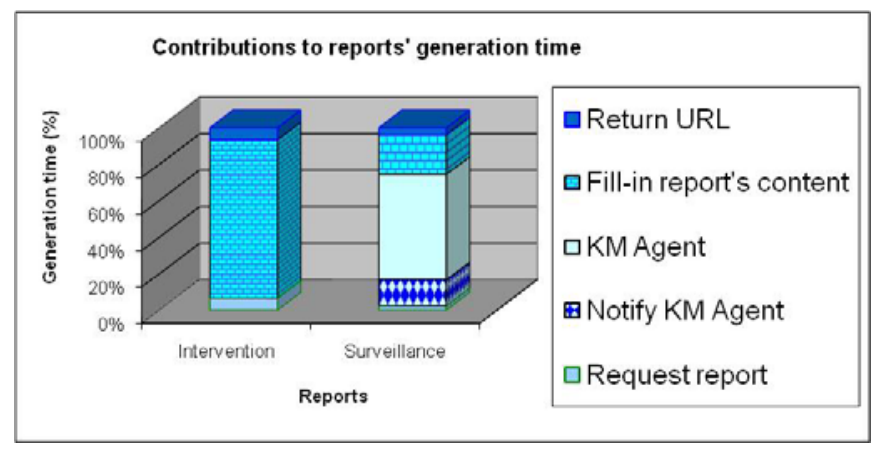

Fig. 4 Contributions to reports' generation time

\section{CONCLUSIONS}

The developed tool allows the evaluation of Telemedicine Systems from the clinical point of view and the technical one: patients and doctors receive feedback that helps them in the daily disease management; and system managers can follow the usage evolution and can detect possible system weaknesses. The tool allows the generation of large reports in real time synthesizing the information in graphics and tables, easing the analysis to users. Furthermore, the use of electronic reports supposes a reduction in time and space in opposition to paper reports.

For Intervention Reports the predominant contribution to time generation is the time for filling the content of the reports and for Surveillance Reports the predominant contribution is the data processing time in KM Agent. These are the two processes that should be optimized to decrease reports' generation time.

The final conclusion is that the use of a Web Service approach allows multiplatform use of the developed electronic report service and the modularity followed in the implementation enables easy system evolution and scalability.

\section{ACKNOWLEDGMENT}

This work was partially supported by a Spanish FIS grant from the Ministry of Health and Consumer Affairs “ADVISING"-FIS PI060437 funded by Health Institute Carlos III.

\section{REFERENCES}

1. Andreassen S., Gómez E., Carson E.R. (2002). Computers in Diabetes 2000. Computer Methods and Programs in Biomedicine, 69 (2).

2. Wang S.J., Middleton B., Prosser L.A., Bardon C.G., Spurr C.D., Carchidi P.J., Kittler A., Goldszer R., Fairchild D., Sussman A., Kuperman G., Bates D. (2003) A cost-benefit analysis of electronic medical records in primary care. American Journal of Medicine 114:397-403.

3. Frénot S., Laforest F. (1999) Medical Record Management Systems: Criticisms and new Perspectives. Methods of Information In Medicine 38:89-95.

4. Wang M., Lau C., Matsen F.A., Kim Y. (2004) Personal Health Information Management System and its Application in Referral Management. IEEE Transactions on Information Technology In Biomedicine 8:287-297.

5. Gómez E.J., Hernando M.E., Vering T., Rigla M., Bott O., GarcíaSáez G., Pretschner P., Brugués E., Schnell O., Patte C., Bergmann J., Dudde R., de Leiva A.(2008) The INCA system: a further step towards a Telemedical Artificial Pancreas. IEEE Transactions on Information Technology in Biomedicine, 12(4):470-479.

6. World Health Organization (2006) Fact sheet $\mathrm{N}^{\mathrm{o}} 312$

7. Rigla M., Hernando M.E., Gómez E.J., Brugués E., García-Sáez G., Capel I., Pons B., de Leiva A., (2008) Real-Time Continuous Glucose Monitoring together with Telemedical Assistance Improve Glycemic Control and Glucose Stability in Pump-Treated Patients. Diabetes Technology \& Therapeutics. 10(3):194-199.

8. Web Consortium, Web Services Activity in W3C (2007) at http://www.w3.org/2002/ws/

9. García-Sáez G., Hernando M.E., Rigla M., Molero J., Capel I., Martínez-Sarriegui I., de Leiva A., Gómez E.J. (2008) Automatic Alarms to detect anomalous patterns in continuous glucose measurements with a Telemedicine System for Diabetes Management. 1st Conference on Advanced Technologies \& Treatments for Diabetes Prague, (27th February - 1st March) 